Molecular description of steady supersonic free jets

S. Montero

Citation: Physics of Fluids 29, 096101 (2017); doi: 10.1063/1.5001250

View online: http://dx.doi.org/10.1063/1.5001250

View Table of Contents: http://aip.scitation.org/toc/phf/29/9

Published by the American Institute of Physics

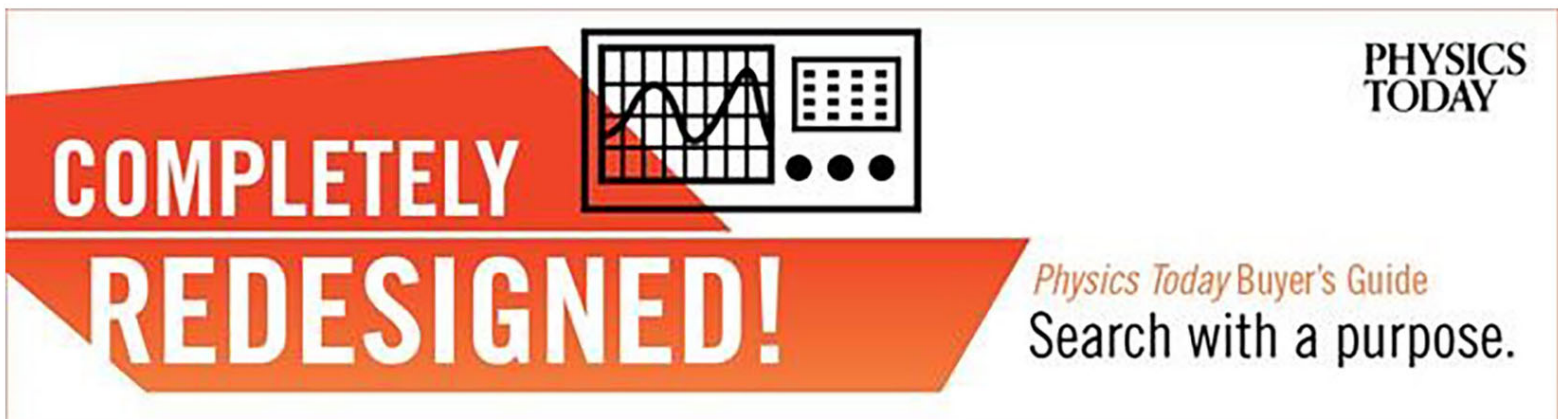




\title{
Molecular description of steady supersonic free jets
}

\author{
S. Montero ${ }^{\mathrm{a})}$ \\ Laboratory of Molecular Fluid Dynamics, Instituto de Estructura de la Materia, CSIC, \\ Serrano 121, 28006 Madrid, Spain
}

(Received 29 May 2017; accepted 18 August 2017; published online 13 September 2017)

\begin{abstract}
A detailed analysis of the non-local thermal equilibrium (n-LTE) problem in the paraxial zone of silence of supersonic free jets is reported. The study is based on a hybrid approach that combines Navier-Stokes equations with a kinetic equation derived from the generalized Boltzmann (WaldmannSnider) equation. The resulting system is solved for those flow quantities not easily amenable to experimental measure (translational temperature, flow velocity, and entropy) in terms of the quantities that can be measured accurately (distance, number density, population of rotational states, and their gradients). The reported solutions are essentially exact and are formulated in terms of macroscopic quantities, as well as in terms of elementary collision processes. Emphasis is made on the influence of dissipative effects onto the flow (viscous and diabatic) and of the breakdown of thermal equilibrium onto the evolution of entropy and translational temperature. The influence of inelastic collisions onto these effects is analysed in depth. The reported equations are aimed at optimizing the experimental knowledge of the n-LTE problem and its quantitative interpretation in terms of state-to-state rates for inelastic collisions. Published by AIP Publishing. [http://dx.doi.org/10.1063/1.5001250]
\end{abstract}

\section{INTRODUCTION}

The study of the molecular aspects of hypersonic flow, shock waves, interstellar media, planetary atmospheres, comets, and many other scientific and technical issues demands a deep understanding of the non-local thermal equilibrium (n-LTE) problem in rarefied gas dynamics. Supersonic free jets and molecular beams have often been employed as a reference system for the study of the relaxation effects associated with n-LTE. ${ }^{1-5}$ Indeed, supersonic free jets produced in the laboratory provide a unique tool for the experimental study of the inelastic and reactive collisions that account for the n-LTE phenomenology. While the macroscopic aspects of relaxation in supersonic jets are relatively well established, ${ }^{6-22}$ its connection to the collision processes is still poorly understood. In general, relaxation has been treated so far in terms of effective relaxation time $\tau$ and collision number $Z$, with little or no relation to the elementary state-to-state collision processes. Since these can be understood and calculated today accurately in the frame of quantum scattering theory, it is time to consider them in some detail. The present work is aimed at this issue, especially in the theory-experiment interphase, with emphasis in the state-to-state processes.

Collisions are the fundamental processes that account for the transfer of energy between molecules in a gas medium, determining its time-space evolution. The Boltzmann equation (BE),

$$
\partial f_{i} / \partial t+\mathbf{v} \cdot \nabla f_{i}=\left(\delta f_{i} / \delta t\right)_{c o l l},
$$

with the generalized (Waldmann-Snider) collision integral (GCI) $\left(\delta f_{i} / \delta t\right)_{\text {coll }^{23-26}}$ explicitly takes into account collisions and provides a molecular description of gas-dynamic systems, in particular supersonic jets. Its practical implementation and

\footnotetext{
a)Electronic mail: emsalvador@iem.cfmac.csic.es
}

connection with experiment is, however, hardly affordable to date since none of the quantities in BE (1) can be determined straight from experiment; $f_{i}(\mathbf{r}, \mathbf{v}, t)$ is the distribution function of the velocity $\mathbf{v}$ for molecules in quantum state $i$ at the position $\mathbf{r}$ and time instant $t$. The left-hand term is due to geometrical effects along the time between collisions, while the right-hand term refers to the changes of $f_{i}$ due to the collisions. Its explicit form reads ${ }^{25}$

$$
\begin{aligned}
\left(\frac{\delta f_{i}\left(\mathbf{r}, \mathbf{v}_{1}, t\right)}{\delta t}\right)_{\text {coll }}= & \sum_{j l m} \iint\left[f_{\ell}\left(\mathbf{r}, \mathbf{v}_{2}^{\prime}, t\right) f_{m}\left(\mathbf{r}, \mathbf{v}_{1}^{\prime}, t\right) \frac{g_{i} g_{j}}{g_{\ell} g_{m}}\right. \\
& \left.-f_{i}\left(\mathbf{r}, \mathbf{v}_{2}, t\right) f_{j}\left(\mathbf{r}, \mathbf{v}_{1}, t\right)\right] \\
& \times\left|\mathbf{v}_{1}-\mathbf{v}_{2}\right| \sigma_{i j \rightarrow \ell m} d \mathbf{e}_{1}^{\prime} d \mathbf{v}_{2},
\end{aligned}
$$

where $\mathbf{v}_{1}, \mathbf{v}_{2}$, and $\mathbf{v}_{1}^{\prime}, \mathbf{v}_{2}^{\prime}$ are, respectively, the pre- and postcollision velocities of collision partners 1 and $2 ; \mathbf{e}_{1}^{\prime}=\mathbf{v}_{1}^{\prime} /\left|\mathbf{v}_{1}^{\prime}\right|$ is a unit vector. Indices $i, j$ and $\ell, m$ label, respectively, the pre- and post-collision quantum states of degeneracy $g_{i}=2 J_{i}$ $+1, g_{j}=2 J_{j}+1, g_{\ell}=2 J_{\ell}+1, g_{m}=2 J_{m}+1$, where $J$ is the angular momentum rotational quantum number; $\sigma_{i j \rightarrow \ell m}(E)$ $=\sigma_{i j \rightarrow \ell m}\left(\chi,\left|\mathrm{v}_{1}-\mathrm{v}_{2}\right|\right)$ is the averaged cross section at a collision deflection angle $\chi$ for the $i j \rightarrow \ell m$ collision event at the kinetic energy $E=\mu\left|\mathbf{v}_{2}-\mathbf{v}_{1}\right|^{2} / 2$, where $\mu$ is the reduced mass of partners 1 and 2 .

Equations (1) and (2) provide the basis for the macroscopic gas-dynamic equations and for the molecular transport coefficients appearing in them, like shear and bulk viscosities, thermal conductivity, and diffusion coefficient. These coefficients have been formulated in terms of the $\sigma_{i j \rightarrow \ell m}$ cross sections for elastic and inelastic collision processes. ${ }^{24,27-30}$ The formulation has been extended to plasma media ${ }^{31}$ and to high-temperature n-LTE problems. ${ }^{32}$ In summary, the literature on the theoretical aspects of BE (1) and GCI (2) is 
rich, though its connection to accurate experiments is far less developed.

Concerning n-LTE experiments in supersonic jets, a major difficulty when dealing with the GCI (2) arises from the stateto-state cross sections $\sigma_{i j \rightarrow \ell m}$, which depend on the collision energy. This quantity is hardly accessible in supersonic jet experiments and, furthermore, not easily manageable from the analytical or numerical point of view. The $\sigma_{i j \rightarrow \ell m} \mathrm{~s}$ can be transformed, however, into state-to-state collision rates (stsrates, in short) which only depend on the local translational temperature, a far more friendly quantity in supersonic jet experiments.

An alternative procedure to the practical limitations of $\mathrm{BE}$ (1) is the Navier-Stokes (NS) equations, which can be derived from it by well-known procedures. However, the NS-equations do not consider explicitly the collision processes, just employing macroscopic quantities such as temperature, pressure, and flow velocity, or combinations of them, jointly with the transport coefficients. Although useful and affordable thanks to the many NS-computational codes available, the NS-equations do not allow for an insight into the elementary state-to-state collision processes involved in the n-LTE problem. To overcome this limitation, the NS-equations must be complemented with a kinetic master equation explicitly expressed in terms of elementary collision processes, as done below. This kinetic master equation, which derives from BE (1) and GCI (2), ${ }^{26}$ is developed below in detail. It allows the quantification of the effect of elementary collision processes onto the flow variables of supersonic free jets.

For all molecular and atomic neutral species, stationary free jets ${ }^{33}$ expanded through an orifice of diameter $D$ into a low-pressure chamber produce a complex gas-dynamic structure that shares a common pattern and many characteristics for a wide range of stagnation conditions. ${ }^{17,19,22,34}$ This gasdynamic structure displays a supersonic/hypersonic core of laminar flow usually referred to as the "zone of silence" of the jet. This region is isolated from the background gas by a normal shock and a lateral barrel of compressed gas and provides an ideal medium for studying the underlying physics of n-LTE on a molecular scale. The zone of silence displays, however, considerable differences from jet to jet, depending not only on the stagnation conditions but also on the nature of the expanded gas. The molecular mass, inertia moment, and intermolecular potential of colliding partners in its entire complexity are responsible for such differences.

In the initial part of the expansion, say, along the first ten orifice diameters of the zone of silence, the temperature drops by more than one order of magnitude, the number density by more than two and the pressure by more than three with respect to stagnation conditions. Simultaneously, the flow velocity increases up to a factor three and the Mach number by one order of magnitude or more with respect to the orifice exit conditions. Moreover, the equilibrium between translational and internal degrees of freedom proper of a bulk gas is destroyed in the expansion, and the distribution of populations of molecular energy levels departs more or less sharply from a Boltzmann distribution, ${ }^{35}$ originating the n-LTE conditions.

The paucity of accurate experimental data on supersonic flow must be emphasized. Though the literature is rich on the subject, most of the fundamental experiments were conducted on wind tunnels, ${ }^{9,36}$ shock tubes, ${ }^{37}$ or molecular beams. ${ }^{6,8,14,15,17}$ Wind tunnel experiments did provide very limited information at the microscopic level and mostly on $\mathrm{N}_{2}$, air, helium or argon species, or their mixtures. At the other end, the molecular beam experiments are conducted at large distances from the orifice, downstream from skimmers, where only terminal flow conditions are observable. There, the supersonic flow is usually frozen, and the information that can be retrieved on the evolution of $n$-LTE is indeed poor. An improvement to this state of things arises from the recently developed supersonic expansion chambers equipped with high space and time resolution diagnostics by Raman spectroscopy. ${ }^{38,39}$ They allow for accurate quantitative measurements in the near field and middle field flow for a wide range of molecular species, as described in the Appendix. The present work is aimed at a more profitable and complete use of experimental data than has been possible so far. Consequently, the main purposes are

- to provide a set of accurate equations for those flow quantities hardly amenable to laboratory measurements (translational temperature, flow velocity, and entropy) so that they can be obtained from other quantities that can be measured accurately (distance to orifice, local number density, populations of quantum states, and their gradients) and

- to provide a quantitative description of the flow in terms of molecular properties (energies of quantum states) and elementary collision processes (state-tostate inelastic collision rates) within the philosophy of the Boltzmann equation.

These goals make unavoidable the use of BE (1) and GCI (2). To this purpose, a hybrid procedure combining the NSequations with the GCI (2) is proposed here in order to link the flow equations with the jet experiment, both at macroscopic scale as well as in collisional terms. The procedure starts from the NS-equations by expressing the flow enthalpy in molecular terms, describing then its evolution due to collisions by means of a kinetic master equation (MEQ). This MEQ derives from BE (1) by transforming the GCI (2) from the domain of energies into the domain of temperatures. ${ }^{26,40}$ The discrete nature of molecular quantum levels is introduced rigorously in this way. This allows overcoming the limitation of some gas-dynamic concepts and procedures based on macroscopic approaches, which often ignore the effects derived from the discrete nature of the internal degrees of freedom.

In order to simplify the mathematics of the discussion as far as possible but preserving the fundamental physics of $\mathrm{n}$-LTE, the discussion is constrained to (1) the thermal domain where vibrational excitation is negligible, (2) single molecule expanding gas, and (3) the paraxial region of the zone of silence of the jet, which is treated here as a quasi-one-dimensional problem closely related to real experiments.

This paper is organized as follows: in Sec. II the startingpoint equations and its range of applicability are presented and discussed. In Sec. III, the formulation of translational temperature as a function of number density, populations of quantum states, and dissipative effects is derived. In Sec. IV, the relation between translational temperature, entropy, and 
dissipative effects is discussed. The link between the macroscopic flow quantities and the inelastic collision processes is presented in Sec. V. The paper is closed with a summary of results and comments. The state-of-the-art on measurements of the primary experimental quantities (distance to orifice, number densities, rotational populations, and their gradients) along the zone of silence of supersonic jets is described briefly in the Appendix.

\section{GOVERNING EQUATIONS}

The following set of conservative equations provides the starting point for the present study concerning the paraxial region of the zone of silence in steady supersonic free jets:

$$
\frac{d}{d z}(\rho v A)=0, \quad \text { Continuity }
$$

$$
\frac{d}{d z}\left(\rho v^{2}+p\right)=d_{1}, \quad \text { Linear Momentum Conservation }
$$

$$
\frac{d}{d z}\left(v\left(\rho e_{t o t}+p\right)\right)=d_{2}, \quad \text { Energy Conservation }
$$

$\frac{d P_{i}}{d t}=n \sum_{j l m}\left(-P_{i} P_{j} k_{i j \rightarrow l m}+P_{\ell} P_{m} k_{\ell m \rightarrow i j}\right)$. Master Equation

All four equations can be derived from the Boltzmann equation (1) with the generalized collision integral (2). Equations (3)-(5) are the well-known NS-equations ${ }^{41}$ constrained to the one-dimensional case, which is a good approximation for the paraxial flow; Eq. (6) is the kinetic master equation. ${ }^{26,40}$ The local quantities in Eqs. (3)-(6) and below are

$z=$ axial distance from the orifice exit plane to a point located on the jet axis,

$\rho(z)=$ density of the gas,

$v(z)=$ flow velocity,

$A=$ constant cross section area for a paraxial stream tube,

$p(z)=$ pressure,

$e_{t o t}(z)=$ total flow energy per mass unit,

$d_{1}(z)$ and $d_{2}(z)=$ dissipative terms, ${ }^{42}$

$P_{i}(z)=$ population probability (population, in short) of a molecular rotational quantum state $i$,

$n(z)=$ number density,

$\mathcal{N}(z)=$ molar number density,

$T_{r}^{\text {eff }}(z)=$ effective rotational temperature,

$k_{i j \rightarrow \ell m}\left(T_{t}\right)=$ sts-rates at the local translational temperature $T_{t}(z)$ for the elementary collision process,

$$
\mathrm{M}_{1}(i)+\mathrm{M}_{2}(j) \longrightarrow \mathrm{M}_{1}(\ell)+\mathrm{M}_{2}(m),
$$

between molecules $\mathrm{M}_{1}$ and $\mathrm{M}_{2}$ undergoing a collision-induced transition from pre-collision quantum states $i$ and $j$ to postcollision states $\ell$ and $m$. The sts-rates are related to the sts-cross sections of the collision integral (2) by the weighted average of the cross sections over a range of energies

$$
k_{i j \rightarrow l m}\left(T_{t}\right)=\frac{\left\langle v_{c}\right\rangle}{\left(k_{B} T_{t}\right)^{2}} \int_{E_{s}}^{\infty} \sigma_{i j \rightarrow l m}(E) e^{\left(-\frac{E}{k_{B} T_{t}}\right)} E d E,
$$

allowing for the transformation from the domain of collision energy $E$ into the domain of an average translational temperature $T_{t}=\left(2 T_{\perp}+T_{\|}\right) / 3$, which is a better suited quantity for describing jet experiments; $\left\langle v_{c}\right\rangle=\left(8 k_{B} T_{t} / \pi \mu\right)^{1 / 2}$ is the average collision velocity of the colliding partners of reduced mass $\mu$, and $E_{s}$ is the minimum energy required for the process (7) to occur. The quantity $E=E_{\text {total }}-E_{i}-E_{j}$ is the available precollision kinetic energy for the $i$ and $j$ rotational levels of the colliding partners. ${ }^{26,40}$ The sts-rates obey the detailed balance relation,

$$
k_{\ell m \rightarrow i j}=k_{i j \rightarrow \ell m} \frac{g_{i} g_{j}}{g_{\ell} g_{m}} e^{\left(E_{\ell}+E_{m}-E_{i}-E_{j}\right) / k_{B} T_{t}},
$$

where $E_{\ell}, E_{m}, E_{i}$, and $E_{j}$ are the energies of the molecular quantum levels. Populations $P_{i}$ and translational temperature $T_{t}$ are related in the zone of silence of the jet through the distribution functions of velocity,

$$
f_{i}(\mathbf{r}, \mathbf{v})=P_{i} n\left(\frac{m}{2 \pi k_{B} T_{t}}\right)^{3 / 2} \exp \left(-\frac{m|\mathrm{C}|^{2}}{2 k_{B} T_{t}}\right),
$$

of Boltzmann equation (1), where $m$ is the molecular mass and $\mathrm{C}$ the peculiar velocity vector (molecule velocity minus flow velocity).

The MEQ (6) describes the time evolution of rotational populations along a jet subject to neither radiation emission nor absorption. It applies wherever three-body collisions are negligible. For consistency with the collision integral (2), the form given here corresponds to a gas of identical but discernible molecules. For jets of identical indiscernible molecules, ${ }^{40}$ some constraints must be imposed to the indices in the sum of MEQ (6).

Other relations employed for later convenience are

$$
\rho=m n=W \mathcal{N},
$$

where molecular mass $m$ (in $\mathrm{kg}$ ) and number density $n$ (in $\mathrm{m}^{-3}$ ) are related to molar mass $W$ (in $\mathrm{kg} / \mathrm{mol}$ ) and molar number density $\mathcal{N}$ (in $\mathrm{mol} \mathrm{m}^{-3}$ ). Pressure is given by

$$
p=n k_{B} T_{t}=\mathcal{N} R T_{t},
$$

where $R=8.31451 \mathrm{~J} \mathrm{~K}^{-1} \mathrm{~mol}^{-1}$ is the universal gas constant. Furthermore, the $\left(\rho e_{t o t}+p\right)$ term in Eq. (5) will be expressed for the present purpose as ${ }^{42}$

$$
\left(\rho e_{t o t}+p\right)=\mathcal{N}\left(H+W \frac{v^{2}}{2}\right)
$$

in terms of the molar enthalpy $(\mathrm{J} / \mathrm{mol})$

$$
H=\frac{5}{2} R T_{t}+E_{r}+E_{v},
$$

where $E_{r}$ and $E_{v}$ are the rotational and vibrational internal energies per mole.

The present work is not aimed at solving Eqs. (3)-(6) but to reduce them to a suitable form for the interpretation of the physics of the jet in connection with those experimental data that can be measured accurately in the laboratory according to the state-of-the-art described in the Appendix. Such data are distance $z$, the local number density $n(z)$ [or $\mathcal{N}(z)$ ], the rotational populations $P_{i}(z)$ (in favorable cases, vibrational populations also), and the population gradients $d P_{i}(z) / d z$ along the jet. For this purpose, Eqs. (4) and (5) are rewritten as

$$
v \frac{d v}{d z}+\frac{1}{\mathcal{N} W} \frac{d p}{d z}=\frac{d_{1}}{\mathcal{N} W},
$$




$$
v \frac{d v}{d z}+\frac{1}{W} \frac{d H}{d z}=\frac{d_{2}}{v \mathcal{N} W} .
$$

Its difference leads to the equation of gradients

$$
\frac{d H}{d z}-\frac{1}{\mathcal{N}} \frac{d p}{d z}=\mathcal{D}
$$

which, together with the MEQ (6), provides a substantial basis to understand in depth the physics of the jet in macroscopic, as well as in molecular terms, allowing for an accurate quantification. The dissipation

$$
\mathcal{D}=\frac{d_{2}}{v \mathcal{N}}-\frac{d_{1}}{\mathcal{N}}
$$

can be expressed as a function of transport coefficients as ${ }^{42}$

$$
\mathcal{D}=\frac{1}{\mathcal{N} v}\left[\left(\frac{d v}{d z}\right)^{2}\left(\frac{4}{3} \mu_{S}+\eta_{V}\right)+\lambda \frac{d^{2} T_{t}}{d z^{2}}+\frac{d \lambda}{d T_{t}}\left(\frac{d T_{t}}{d z}\right)^{2}\right],
$$

where $\mu_{S}$ is the shear viscosity, $\eta_{V}$ is the volume viscosity, and $\lambda$ is the heat conductivity.

For expanding species other than helium, ${ }^{43,44}$ the flow variables not amenable to accurate measurements in laboratory jets are the translational temperature $T_{t}$, the flow velocity $v$, and the entropy $S$. As shown below, these quantities can be inferred from Eqs. (14) and (17). The molecular interpretation in terms of collisions follows from combinations with the MEQ (6).

At this point it is pertinent to establish the range of applicability of the equations given above, taking into account the characteristics of the flow and the spatial domain of the jet where accurate measurement of the primary variables $z, n(z)$, $P_{i}(z)$, and $d P_{i}(z) / d z$ is possible (see Appendix). For free jets expanded through orifices of diameter $D$ in the range between 50 and $500 \mu \mathrm{m}$, recent experimental developments allow for such measurements from $z \approx 0.15 D$ up to $z \approx 20 D$, depending on the nature of the expanded gas and on the stagnation conditions. Two parameters are useful to estimate the applicability of the equations derived here, namely, the Reynolds number $\operatorname{Re}_{e}=\rho v D / \mu_{S}$ at exit flow values and the local Knudsen number $\operatorname{Kn}(z)$.

The validity of the results reported below depends on the following factors:

- Jet stagnation conditions such that condensation along the expansion is negligible.

- Range of validity of Eqs. (3)-(5) under the constraint $T_{t}=\left(2 T_{\perp}+T_{\|}\right) / 3$. This is tentatively estimated by $\mathrm{Kn}(z)<0.2$ values of the local Knudsen number. ${ }^{45,46}$

- Small non-equilibrium for the parallel and perpendicular translational temperatures, i.e., $T_{\|} \approx T_{\perp}$. As a guide, the limit $z \leq 20 D$ holds, but this depends on $\operatorname{Kn}(z)$ and $\operatorname{Re}_{e}$ values.

- Exit Reynolds number $\operatorname{Re}_{e}$ large enough to circumvent the invasion of the paraxial region by molecules from the detached boundary layer, and from the jet barrel and normal shock boundaries. A tentative limit is $\operatorname{Re}_{e} \geq$ 200, but smaller values might also hold. Such limits imply a weak to moderate dissipative term $\mathcal{D}$.
The local Knudsen number along the jet is defined as ${ }^{46}$

$$
\mathrm{Kn}(z)=\frac{\Lambda}{n}\left|\frac{d n}{d z}\right|
$$

where

$$
\Lambda=\left(\sqrt{2} n \pi d^{2}\right)^{-1}
$$

is the local mean free path for a molecule of collision diameter $d$. Since the number density decays for $z / D>1$ as $n(z) \propto$ $n_{0}(D / z)^{2}$, the local Knudsen number can be expressed as

$$
\mathrm{Kn}(z)=\frac{c(\gamma) z}{n_{0} d^{2} D^{2}}
$$

where $c(\gamma)$ is a constant that only depends on the heat capacity ratio $\gamma=C_{p} / C_{V}$ of the expanded species; as a guide, $c(\gamma) \approx$ 4.1 for $\gamma=7 / 5$ and $c(\gamma) \approx 2.8$ for $\gamma=5 / 3$. Imposing the $\mathrm{Kn}<0.2$ limit one obtains

$$
z / D<0.2 n_{0} D d^{2} / c(\gamma)
$$

as the $z$-domain of applicability of the equations derived in the present work as a function of stagnation conditions, orifice diameter, and expanded species. Somewhat different validity limits can be derived from criteria not based on the local Knudsen number for the continuum-transition flow regime. ${ }^{47}$

It must be emphasized that some quantities widely employed for the description of supersonic free jets are often ill-defined and may bias the quantitative interpretation of the jets at low $\operatorname{Re}_{e}$ values. Therefore, they will be circumvented here as far as possible. These quantities are the rotational temperature $T_{r}$ (vibrational temperature $T_{v}$ also), the rotational heat capacity $C_{r}=d H / d T_{r}$, and the heat capacity ratio $\gamma$. The background reason is that the supersonic jets are nonequilibrium systems where the distribution of populations $P_{i}$ always departs progressively from a Boltzmann distribution for increasing energies of the rotational levels. ${ }^{35}$ This effect is mild in $\mathrm{N}_{2}, \mathrm{O}_{2}$, and heavier molecules jets at $\mathrm{Re}_{e}>200$. In this case an effective rotational temperature $T_{r}^{e f f}$ can be a suited quantity for most purposes. On the contrary, jets of lighter molecules, even at $\operatorname{Re}_{e}>200$ cannot be accurately described in terms of rotational temperature. The most extreme cases are $\mathrm{H}_{2}$ jets, where the departure of rotational populations $P_{i}$ from a Boltzmann distribution is dramatic even at $\operatorname{Re}_{e}>10000$.

For the above-given reasons, the present work is preferably formulated in terms of rotational populations $P_{i}$. This formulation is completely general and valid for jets of heavy as well of light molecules, regardless of the energy transfer efficiency between rotation and translation degrees of freedom. However, some equations will also be given in terms of a single effective rotational temperature, $T_{r}^{e f f}$, defined in terms of population ratios of the lowest rotational energy levels. This simplifies in favorable cases the use of a number of rotational populations $P_{i}$ if the conditions of the jet are such that the $T_{r}^{e f f}$ concept applies with little loss of accuracy.

The $z \leq 20 D, \mathrm{Kn}<0.2$, and $\mathrm{Re}_{e} \geq 200$ limit span variations of up to three orders of magnitude of $n(z)$ and more than one order of magnitude of $T_{t}(z)$, allowing for accurate molecular descriptions of the jet in these ranges by means of the equations given below. 


\section{RELATIONSHIP BETWEEN NUMBER DENSITY, TRANSLATIONAL TEMPERATURE, AND ROTATIONAL POPULATIONS}

The relation between the flow variables $\mathcal{N}(z), T_{t}(z), v(z)$, and $P_{i}(z)$ in the free jet is not simple. A highly simplified relation between $\mathcal{N}$ and $T_{t}$, ignoring $P_{i}$ and the n-LTE state of the jet, is given by the isentropic approximation for a perfect gas. It is summarized next by the popular (but inaccurate) relations in terms of the Mach number $M,{ }^{9,13,17}$

$$
\mathcal{N}=\mathcal{N}_{0}\left(1+M^{2}(\gamma-1) / 2\right)^{\frac{1}{1-\gamma}},
$$

and

$$
T_{t}=T_{0}\left(1+M^{2}(\gamma-1) / 2\right)^{-1},
$$

which satisfy the isentropic relation

$$
T_{t} \mathcal{N}^{\gamma-1}=\text { constant. }
$$

In this approximation, the flow velocity is given by

$$
v=M \sqrt{\frac{\gamma R T_{0}}{W}}\left(1+M^{2}(\gamma-1) / 2\right)^{-1 / 2} .
$$

Solving Euler's equations by the method of characteristics for the perfect gas, the Mach number for an axisymmetric jet can be expressed as a function,

$$
M=\tilde{z}^{\gamma-1}\left(b+c / \tilde{z}+d / \tilde{z}^{2}+e / \tilde{z}^{3}\right),
$$

of the reduced distance $\tilde{z}=z / D$ and parameters $b, c, d, e$. These parameters depend on $\gamma$ and have been established empirically for $\tilde{z} \geq 1.9,17,38$

Though useful for some purposes, the isentropic approximation given above is poor from the physical point of view, misleading in several aspects, and often producing inaccurate results. Accurate relations are derived here starting from Eq. (17) of gradients, taking into account that the molar enthalpy (14) is accurately expressed by

$$
H=H_{t}+H_{r}=\frac{5}{2} R T_{t}+R \sum_{i} \epsilon_{i} P_{i},
$$

in terms of the actual populations $P_{i}$ regardless of whether or not they obey a Boltzmann distribution. This circumvents the use of concepts that are ill-defined in the jet, like the rotational temperature and the rotational heat capacity; $\epsilon_{i}$ is the energy of molecular quantum state $i$ referred to the lowest one, expressed in Kelvin $\left(1 \mathrm{~cm}^{-1}=1.4388 \mathrm{~K}\right)$. In order to simplify the discussion, the vibrational contribution has been ignored in Eq. (29).

Expressing in Eq. (17) the gradient of the molar enthalpy along the jet as

$$
\frac{d H}{d z}=\frac{5}{2} R \frac{d T_{t}}{d z}+R \sum_{i} \epsilon_{i} \frac{d P_{i}}{d z},
$$

one arrives at the differential equation

$$
\frac{d T_{t}}{d z}+p(z) T_{t}=q(z)
$$

where

$$
p(z)=-\frac{2}{3 \mathcal{N}} \frac{d \mathcal{N}}{d z},
$$

and

$$
q(z)=\frac{2}{3 R}\left[\mathcal{D}-R \sum_{i} \epsilon_{i} \frac{d P_{i}}{d z}\right] .
$$

Equation (31) is non-linear due to the dissipative term $\mathcal{D}$, which depends on $\mathcal{N}, v, T_{t}$, and on additional derivatives according to (19). However, within the limits described above, the equation can be linearized perturbatively to a good approximation by assuming in a first step the inviscid-adiabatic approximation $(\mathcal{D}=0)$. This leads to the inviscid-adiabatic $(i a)$ solution,

$T_{t}^{i a}(z)=\left(\frac{\mathcal{N}}{\mathcal{N}_{0}}\right)^{2 / 3}\left[T_{0}+\frac{2}{3 R} \int_{z_{0}}^{z}\left(\frac{\mathcal{N}}{\mathcal{N}_{0}}\right)^{-2 / 3}\left(-R \sum_{i} \epsilon_{i} \frac{d P_{i}}{d z}\right) d z\right]$,

for the translational temperature; $T_{0}$ and $\mathcal{N}_{0}$ are the stagnation temperature and number density of the jet, respectively, and $z_{0}$ is a point upstream enough from the orifice where $T_{0}$ and $\mathcal{N}_{0}$ hold. Since $\mathcal{N}$ and $d P_{i} / d z$ are experimental quantities, an explicit zero order dissipation $\mathcal{D}^{(0)}(z)$ can be calculated from Eq. (19) taking into account that in the inviscid-adiabatic approximation $(\mathcal{D}=0)$ the flow velocity is

$$
v^{i a}(z)=\sqrt{\frac{R}{W}}\left[5\left(T_{0}-T_{t}^{i a}(z)\right)+2 \sum_{i} \epsilon_{i}\left(P_{i 0}-P_{i}(z)\right)\right]^{1 / 2},
$$

where $P_{i 0}$ is the population of rotational state $i$ at the stagnation temperature $T_{0}$. Replacing $\mathcal{D}$ in (33) by

$\mathcal{D}^{(0)}=\frac{1}{\mathcal{N} v^{i a}}\left[\left(\frac{d v^{i a}}{d z}\right)^{2}\left(\frac{4}{3} \mu_{S}+\eta_{V}\right)+\lambda \frac{d^{2} T_{t}^{i a}}{d z^{2}}+\frac{d \lambda}{d T_{t}^{i a}}\left(\frac{d T_{t}^{i a}}{d z}\right)^{2}\right]$,

with $\mu_{S}, \eta_{V}$, and $\lambda$ at the temperature $T_{t}^{i a}, \mathcal{D}^{(0)}$ can be expressed as a function of $z$, Eq. (31) becoming linear. Its solution is

$$
\begin{aligned}
T_{t}(z)= & T_{0}\left(\frac{\mathcal{N}}{\mathcal{N}_{0}}\right)^{2 / 3}\left[1+\frac{2}{3 R T_{0}} \int_{z_{0}}^{z}\left(\frac{\mathcal{N}}{\mathcal{N}_{0}}\right)^{-2 / 3}\right. \\
& \left.\times\left(\mathcal{D}^{(0)}-R \sum_{i} \epsilon_{i} \frac{d P_{i}}{d z}\right) d z\right]
\end{aligned}
$$

where everything in the right-hand side can be obtained from the experiment (see Appendix). Under conditions where the effective rotational temperature is a good approximation, i.e., at mild $\mathrm{n}$-LTE, the above equation reads ${ }^{42}$

$$
\begin{aligned}
T_{t}(z)= & T_{0}\left(\frac{\mathcal{N}}{\mathcal{N}_{0}}\right)^{2 / 3}\left[1+\frac{2}{3 R T_{0}} \int_{z_{0}}^{z}\left(\frac{\mathcal{N}}{\mathcal{N}_{0}}\right)^{-2 / 3}\right. \\
& \left.\times\left(\mathcal{D}^{(0)}-C_{r}^{\text {eff }} \frac{d T_{r}^{\text {eff }}}{d z}\right) d z\right]
\end{aligned}
$$

where $C_{r}^{e f f}=d H_{r} / d T_{r}^{\text {eff }}$ is the effective rotational heat capacity per mole. This way the translational temperature along the jet can be obtained indirectly on an experimental basis by means of Eq. (37) or (38).

Note that Eqs. (37) and (38) can be factorized in the form

$$
T_{t}(z)=T_{t}^{e l} \times(1+F),
$$

where

$$
T_{t}^{e l}(z)=T_{0}\left(\frac{\mathcal{N}}{\mathcal{N}_{0}}\right)^{2 / 3},
$$

is the translational temperature of an ideal monoatomic jet $(\gamma=5 / 3)$, which is governed by just elastic collisions. The term $F$ (always positive) can thus be interpreted as the n-LTE 
perturbation to the ideal $(\gamma=5 / 3)$ jet caused by the dissipation $\mathcal{D}$ and by the rotational degrees of freedom. For negligible dissipation and rotational populations $P_{i}$ nearly obeying a Boltzmann distribution at the effective rotational temperature $T_{r}^{\text {eff }} \approx T_{t}$, Eq. (39) leads to the isentropic relations (25) and (26) with the $\gamma$ factor of the particular molecular species. If these conditions are not satisfied, Eqs. (24)-(27) are not accurate.

\section{ENTROPY ALONG THE JET}

In this section, several aspects of entropy are shown. First, how entropy increment along the jet can be described in terms of dissipation and rotational-translational nonequilibrium or, alternatively, in terms of rotation and translation degrees of freedom. Second, the way is shown how the isentropic temperature can be derived from experiment, and third, how the actual translational temperature relates to the isentropic temperature. Two $\mathrm{H}_{2}$-jets at high and low exit Reynolds number serve to illustrate these three aspects in Fig. 1. Eventually, an alternative procedure to obtain the translational temperature from the experiment by intermediate of entropy is proposed.
Since the flow variables in jets span over orders of magnitude depending on stagnation conditions, nature of the expanded gas, and distance $z$ to the orifice, it is not easy to conclude $a$ priori about the evolution of entropy along a particular jet. Hence, a rigorous discussion on entropy is pertinent.

\section{A. Dissipation and nonequilibrium}

According to a recent work, ${ }^{42}$ the increment of total entropy can be expressed in terms of dissipation $\mathcal{D}$ and of the rotation-translation thermal nonequilibrium, $T_{r}^{e f f} \neq T_{t}$, as

$$
\Delta S_{\text {total }}=\Delta S_{\text {dis }}+\Delta S_{\text {neq }}
$$

where

$$
\Delta S_{d i s}=\int_{z_{0}}^{z} \frac{\mathcal{D}}{T_{t}} d z
$$

and

$$
\Delta S_{n e q}=-\int_{z_{0}}^{z} C_{r}^{e f f}\left(\frac{1}{T_{t}}-\frac{1}{T_{r}^{e f f}}\right)\left(\frac{d T_{r}^{e f f}}{d z}\right) d z .
$$

Both contributions are positive; $\Delta S_{\text {dis }}$ because $\mathcal{D} / T_{t}$ is positive; $\Delta S_{\text {neq }}$ because $T_{r}^{\text {eff }}>T_{t}$ and $d T_{r}^{\text {eff }} / d z<0$ (rotational cooling)
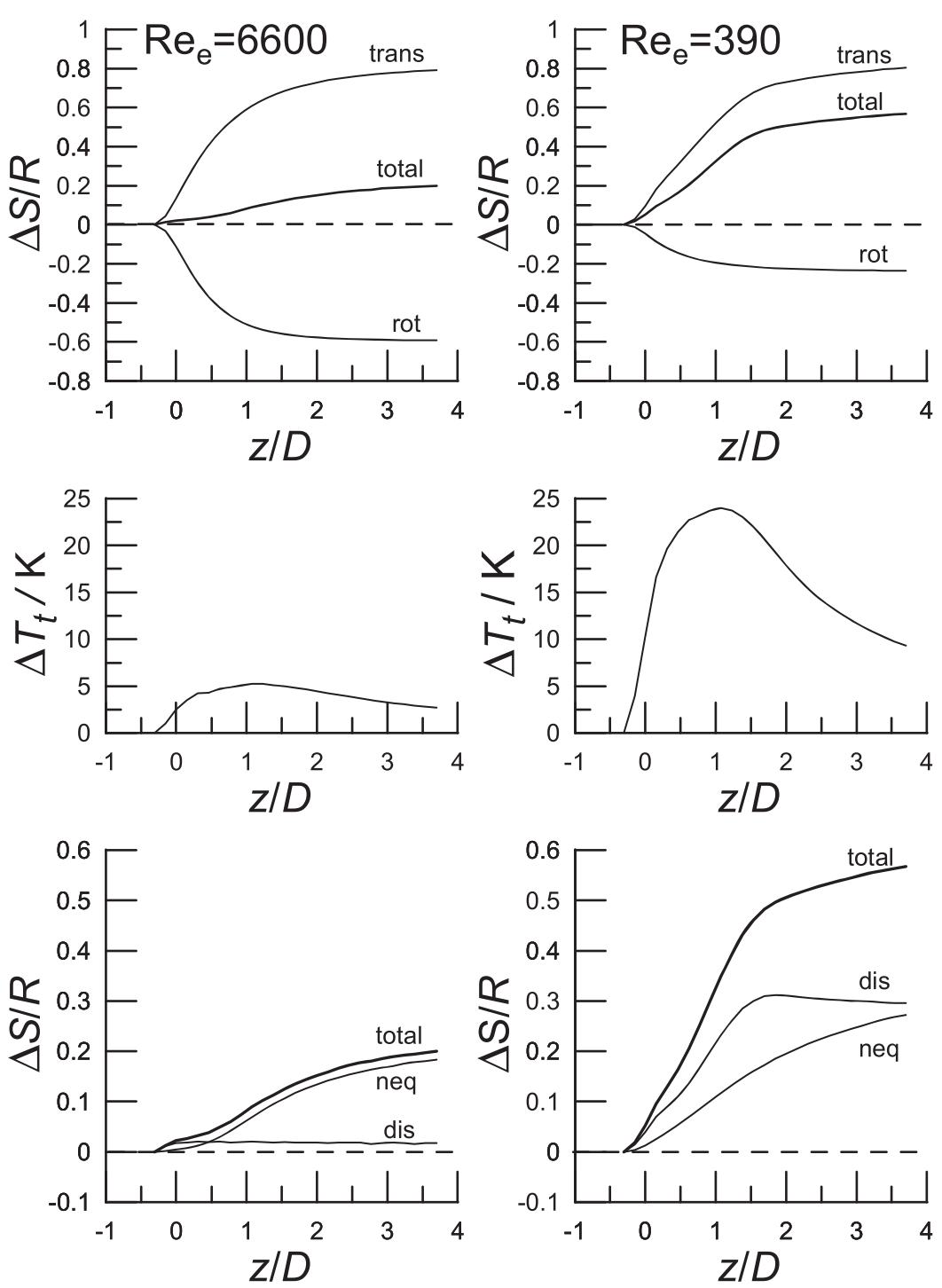

FIG. 1. Entropy and temperature increments along two supersonic jets of $\mathrm{H}_{2}$ at the high (left) and low (right) exit Reynolds number $\mathrm{Re}_{e}$ : (top) in terms of translational and rotational contributions, Eqs. (45)-(52); (bottom) in terms of dissipation and $T_{r}^{e f f} \neq T t$ non-equilibrium, Eqs. (41)-(43); (middle) increments of translational temperature due to the increment of total entropy according to Eq. (57). 
along any real-gas jet. Consequently, $\Delta S_{\text {total }}$ is always positive in supersonic jets of real gases, consistently with the

$$
\Delta S_{\text {total }}>0
$$

condition of irreversible processes.

As an example of this behavior, Fig. 1 (bottom) shows the evolution of entropy along two $\mathrm{H}_{2}$-jets in terms of dissipation, $\Delta S_{\text {dis }}$, and thermal nonequilibrium, $\Delta S_{n e q}$. Both jets were expanded through a $D=325 \mu \mathrm{m}$ orifice at stagnation conditions $p_{0}=3300 \mathrm{mbar}, T_{0}=364 \mathrm{~K}, \mathrm{Re}_{e}=6600$, and $p_{0}=230$ mbar, $T_{0}=295 \mathrm{~K}, \operatorname{Re}_{e}=390$. Note that at high $\operatorname{Re}_{e}$ values (bottom, left), the dissipative contribution is negligible, while most increment of total entropy is due to the $T_{r}^{\text {eff }}>T_{t}$ nonequilibrium. In contrast, at low $\mathrm{Re}_{e}$ values (bottom, right), dissipative and nonequilibrium contributions are large and of comparable magnitude, producing a far larger $\Delta S_{\text {total }}$ entropy increment.

\section{B. The opposite effect of rotation and translation degrees of freedom}

Following now a statistical mechanics approach, let us express additively the molar entropy along the jet in terms of translational $S_{\text {trans }}$ and rotational $S_{\text {rot }}$ contributions as

$$
S_{\text {total }}(z)=S_{\text {trans }}(z)+S_{\text {rot }}(z) .
$$

The translational contribution to the entropy is given by ${ }^{48}$

$$
S_{\text {trans }}(z)=\frac{5}{2} R+R \ln \left(K_{t} \frac{T_{t}^{3 / 2}}{n}\right),
$$

where

$$
K_{t}=\left(\frac{2 \pi m k}{h^{2}}\right)^{3 / 2}
$$

is a constant. In its turn, the rotational contribution to the entropy can be expressed in terms of the rotational populations as $^{49-51}$

$$
S_{r o t}=S_{r o t}^{0}+R \sum_{i} P_{i} \ln \left(\frac{\omega_{i}\left(2 J_{i}+1\right)}{P_{i}}\right),
$$

where $J_{i}$ is the angular momentum quantum number of rotational level $i$, with population $P_{i}$, and statistical weight $\omega_{i}$. According to Ref. 42, $S_{\text {rot }}$ can also be expressed in terms of the effective rotational temperature as

$$
S_{r o t}=C_{r}+\frac{N}{2} R \ln \left(\frac{T_{r}^{e f f}}{\Theta_{r}}\right),
$$

where $C_{r}$ is a constant, $N$ the number of rotational degrees of freedom, and $\Theta_{r}=\beta B$, the characteristic rotational temperature; $B$ is the molecular rotational constant in $\mathrm{cm}^{-1}$ and $\beta=1.4388 \mathrm{~K} / \mathrm{cm}^{-1}$. Equation (49) is less accurate than the "exact" form (48) and is just valid for $T_{r}^{e f f}>\Theta_{r}$. From (49) it follows that the increment of rotational entropy along the jet,

$$
\Delta S_{\text {rot }}=\frac{N R}{2} \ln \left(\frac{T_{r}^{e f f}+\Delta T_{r}^{e f f}}{T_{r}^{e f f}}\right),
$$

is always negative due to the rotational cooling $\left(\Delta T_{r}^{e f f}<0\right)$ along the expansion. As has been shown in Subsection IV A, the total increment of entropy along the jet must be positive. Since $\Delta S_{\text {total }}>0$ and $\Delta S_{\text {rot }}<0$, this implies that the translational contribution $\Delta S_{\text {trans }}$ must be positive and larger than the absolute value of the negative rotational contribution. So, the condition,

$$
\Delta S_{\text {trans }}>\left|\Delta S_{\text {rot }}\right|, \quad \text { with } \quad \Delta S_{\text {rot }}<0,
$$

appears as a characteristic of molecular supersonic jets in the zone of silence. According to (45) and (51), the consequence is that the competition of translational (positive) and rotational (negative) contributions tends to reduce the entropy increment along molecular jets with respect to the entropy of atomic jets of comparable dissipation $\mathcal{D}$. In molecular jets expanded at high $\operatorname{Re}_{e}$ values, this compensation is highly effective leading to quasi-isentropic jets.

A sample of this general behavior in two molecular hydrogen jets generated, respectively, at high and low Reynolds number $\left(\operatorname{Re}_{e}=6600\right.$ and 390) is shown in Fig. 1 (top). At $\operatorname{Re}_{e}=6600,\left|\Delta S_{\text {rot }}\right| \approx \Delta S_{\text {trans }}$ with the strong rot-trans compensation and small increment of entropy shown in Fig. 1 (top, left). In contrast, at $\operatorname{Re}_{e}=390,\left|\Delta S_{\text {rot }}\right|<<\Delta S_{\text {trans }}$, with little rot-trans compensation and higher entropy production, as shown in Fig. 1 (top, right).

\section{Isentropic versus actual translational temperature}

The total increment of entropy along the jet, from stagnation conditions up to a point $z$,

$$
\begin{aligned}
\Delta S_{\text {total }}=\Delta S_{\text {trans }}+\Delta S_{\text {rot }}= & R\left[\ln \left(\frac{T_{t}^{3 / 2} n_{0}}{T_{0}^{3 / 2} n}\right)+\sum_{i} \ln \left(\frac{2 J_{i}+1}{P_{i}}\right)^{P_{i}}\right. \\
& \left.-\sum_{i} \ln \left(\frac{2 J_{i}+1}{P_{i 0}}\right)^{P_{i 0}}\right]
\end{aligned}
$$

is obtained from Eqs. (45)-(48); $T_{0}$ and $n_{0}$ are the stagnation temperature and number density, and $P_{i 0}$ the rotational populations at stagnation temperature $T_{0}$. Statistical weights $\omega_{i}$ have been omitted imposing the normalization condition $\sum_{i} P_{i}=1$ upon the rotational populations.

If the rotational contribution is expressed in terms of effective rotational temperature, one obtains ${ }^{42}$

$$
\Delta S_{\text {total }}=\Delta S_{\text {trans }}+\Delta S_{\text {rot }}=R \ln \left[\left(\frac{T_{t}}{T_{0}}\right)^{3 / 2}\left(\frac{n_{0}}{n}\right)\left(\frac{T_{r}^{e f f}}{T_{0}}\right)^{N / 2}\right] .
$$

Imposing onto (52) and (53) the isentropic condition $\Delta S_{\text {total }}=$ 0 , one obtains the expressions

$$
T_{t}^{i s e n}(z)=T_{0}\left[\left(\frac{n}{n_{0}}\right) C \prod_{i}\left(\frac{P_{i}}{2 J_{i}+1}\right)^{P_{i}}\right]^{\frac{2}{3}},
$$

with constant $C$ given by

$$
C=\prod_{i}\left(\frac{2 J_{i}+1}{P_{i 0}}\right)^{P_{i 0}},
$$

for the isentropic temperature as a function of $n$ and $P_{i}$, and

$$
T_{t}^{i s e n}(z)=T_{0}\left(\frac{n}{n_{0}}\right)^{2 / 3}\left(\frac{T_{0}}{T_{r}^{e f f}}\right)^{N / 3},
$$

as a function of $n$ and $T_{r}^{e f f}$. Since $n, P_{i}$, and $T_{r}^{e f f}$ are experimental quantities, $T_{\text {isen }}$ is also a quantity that can be obtained 
from the experiment with very good accuracy (see Appendix). It serves as a useful reference in jet experiments as well as in numerical calculations of jet flow by means of NS or DSMC (Direct Simulation Monte Carlo) codes.

It must be emphasized that $T_{t}^{\text {isen }}$ is not the true translational temperature $T_{t}$. Inverting Eq. (52) proves that isentropic and translational temperatures are related by the total increment of entropy as

$$
T_{t}(z)=T_{t}^{i s e n}(z) \times e^{\frac{2}{3} \frac{\Delta S_{\text {total }}}{R}} .
$$

Since $\Delta S_{\text {total }}(z)>0$ holds along the jet, as has been shown in Subsection IV A, a major consequence of the increment of entropy along the jet is an increase of the true translational temperature with respect to the isentropic temperature. Consequently, the condition

$$
T_{t}(z)>T_{t}^{i s e n}(z),
$$

always holds for the zone of silence of real supersonic jets.

Figure 1 (middle) shows the increment $\Delta T_{t}=T_{t}-T_{t}^{i s e n}$ of the actual translational temperature with respect to the isentropic temperature due to entropy in two jets at $\operatorname{Re}_{e}=6600$ and $\operatorname{Re}_{e}=390$, with a characteristic maximum at $z / D \approx 1$. This maximum is more pronounced for smaller Reynolds number $\operatorname{Re}_{e}$ at exit.

\section{An alternative procedure for retrieving the translational temperature from experiment via entropy}

Equation (57) also provides a convenient alternative to Eqs. (37) and (38) for a practical determination of the actual translational temperature along the jet. Accordingly, the exponential term $\exp \left(2 \Delta S_{\text {total }} / 3 R\right)$ can be interpreted and calculated as an usually small perturbation to the isentropic temperature. Since $\Delta S_{\text {total }}$ depends itself on $T_{t}$ according to Eqs. (41)-(43), replacing $T_{t}$ by $T_{t}^{i s e n}$, or by the inviscid-adiabatic $T_{t}^{i a}$ of Eq. (34), and $\mathcal{D}$ by $\mathcal{D}^{(0)}$ of Eq. (36) yields a good approximation for jets expanded at not too low $\mathrm{Re}_{e}$. Since the progression,

$$
T_{t}^{\text {isen }}<T_{t}^{i a}<T_{t},
$$

always holds in the zone of silence, a better approximation is obtained employing $T_{t}^{i a}$. This leads to a zero-order approximation of Eq. (41) for the entropic increment,

$$
\Delta S_{\text {total }}^{(0)}(z)=\int_{z_{0}}^{z} \frac{\mathcal{D}^{(0)}}{T_{t}^{i a}} d z-\int_{z_{0}}^{z} C_{r}^{e f f}\left(\frac{1}{T_{t}^{i a}}-\frac{1}{T_{r}^{e f f}}\right)\left(\frac{d T_{r}^{e f f}}{d z}\right) d z,
$$

where all quantities can be obtained from experiment (see Appendix). Imposing $\Delta S_{\text {total }}^{(0)}$ upon Eq. (57) leads to a zeroorder translational temperature $T_{t}^{(0)}$, which is more accurate than the experimental isentropic temperature $T_{t}^{\text {isen }}$. The procedure can be repeated recursively improving the accuracy of $T_{t}$ in each step. The convergence is fast, usually requiring only one cycle. However, for very light molecules like $\mathrm{H}_{2}, \mathrm{HD}$, and $\mathrm{D}_{2}$, two or three cycles are needed at $T_{t}<20 \mathrm{~K}$ to reach numerical accuracy on the order of $0.5 \mathrm{~K}$ for $T_{t}$. With the indicated recursive procedure, Eqs. (57) and (60) provide an increasingly accurate quantification of the entropy and its effect onto the actual translational temperature along the jet with respect to their isentropic and inviscid-adiabatic approximations.

\section{THE MOLECULAR CONNECTION}

The $\Delta S_{\text {total }}, \Delta S_{\text {trans }}, \Delta S_{\text {rot }}, \Delta S_{\text {dis }}$, and $\Delta S_{\text {neq }}$ entropy increments, the $T_{t}, T_{t}^{i s e n}, T_{t}^{i a}$ temperatures, and the experimental $n$, $P_{i}$, and $T_{r}^{e f f}$ quantities strongly depend on the stagnation conditions of the jet $n_{0}$ and $T_{0}$, orifice diameter, and single-molecule properties (mass, energy of internal quantum levels). How the above contributions are related has been shown in Secs. I-IV. But those contributions also depend on two-molecule properties, the inelastic collision sts-rates. This intricate dependence is discussed next.

A merit of Eqs. (37)-(40) and (57)-(63) is the link they provide for the interpretation of the translational and rotational temperatures, as well as of entropy, in terms of molecular collisions in the flow.

Let us start with Eqs. (37)-(39) for the translational temperature. Taking into account the MEQ (6) and the relations,

$$
d P_{i} / d z=\frac{n}{v} \sum_{j l m}\left(-P_{i} P_{j} k_{i j \rightarrow l m}+P_{\ell} P_{m} k_{\ell m \rightarrow i j}\right)
$$

and

$$
\frac{d T_{r}^{e f f}}{d z}=\frac{R}{C_{r}^{e f f}} \sum_{i} \epsilon_{i} \frac{d P_{i}}{d z},
$$

the perturbative factor $F$ in (39) can be rewritten as

$$
\begin{aligned}
F(z)= & \frac{2}{3 R T_{0}} \int_{z_{0}}^{z}\left(\frac{n}{n_{0}}\right)^{-2 / 3}\left(\mathcal{D}^{(0)}-R \frac{n}{v} \sum_{i} \epsilon_{i}\right. \\
& \left.\times \sum_{j l m}\left(-P_{i} P_{j} k_{i j \rightarrow l m}+P_{\ell} P_{m} k_{\ell m \rightarrow i j}\right)\right) d z,
\end{aligned}
$$

showing explicitly the various collisional effects onto the translational temperature $T_{t}$. As inferred from Eqs. (39) and (40), the main contribution to $T_{t}$ is $T_{t}^{e l}$, which is governed by only elastic collisions as corresponds to an ideal $\gamma=5 / 3$ monoatomic jet. The temperature of this reference jet is perturbed by the factor $F$, which includes the effect of elastic as well inelastic contributions according to (63). In $F$ the elastic collisions appear only in the dissipation $\mathcal{D}^{(0)}$. On the other hand, the inelastic collisions contribute in different ways, in part to $\mathcal{D}^{(0)}$ and, specially, governing the effect of the rotational degrees of freedom by means of the $k_{i j \rightarrow l m}$ rates.

In $\mathcal{D}^{(0)}$ the shear viscosity $\mu_{S}$ depends essentially on elastic contributions ${ }^{46,52,53}$ and is a well characterized quantity for many molecular species of gas-dynamic interest. ${ }^{54}$ The heat conductivity $\lambda$ depends on both, elastic and inelastic collisions. Although it has been formulated in terms of state to state cross sections for elementary collision processes, ${ }^{29,30}$ no explicit expressions in terms of $k_{i j \rightarrow l m}$ rates seem to be available. Accurate first principles calculations for $\lambda$ in terms of elementary collision processes have been reported so far only for $\mathrm{N}_{2}{ }^{55}$ and $\mathrm{H}_{2} .{ }^{56}$ Experimental values have been reported for $\mathrm{H}_{2}$ in a wide thermal range ${ }^{57,58}$ and above $77 \mathrm{~K}$ for other molecular species. ${ }^{54}$ The bulk viscosity $\eta_{V}$ exclusively depends on inelastic collisions and has been formulated recently in terms of $k_{i j \rightarrow l m}$ rates. ${ }^{59}$ Very few data have been reported on $\eta_{V}$ with the exception of $\mathrm{H}_{2}, \mathrm{CO}$, and $\mathrm{N}_{2}$ species. ${ }^{30,59-62}$ Guiding values for $\mu_{S}, \lambda$, and $\eta_{V}$ can be obtained from simplified molecular models. ${ }^{31,46,52,53}$ 
The relative importance of $\mu_{S}, \lambda$, and $\eta_{V}$ in $\mathcal{D}^{(0)}$ strongly depends on the particular molecular species. As a general rule, the effect of bulk viscosity is dominant in light species with large rotational constants $\left(B>10 \mathrm{~cm}^{-1}\right)$ and $k_{i j \rightarrow l m}$ rates smaller than $\approx 10^{-17} \mathrm{~m}^{3} \mathrm{~s}^{-1}$, i.e., prone to inefficient transfer of energy between translation and rotation degrees of freedom and to strong n-LTE conditions. A coarse inverse correlation $\mathcal{D} \propto 1 / \operatorname{Re}_{e}$ implies large dissipative and entropic contributions in jets of light molecular species expanded at low stagnation pressure.

On the other hand, if the rotational temperature gradient (62) is expressed in terms of rotational population gradients (61), the explicit dependence of entropy increment on inelastic collisions appears explicitly in

$$
\begin{aligned}
\Delta S^{(0)}(z)= & \int_{z_{0}}^{z} \frac{\mathcal{D}^{(0)}}{T_{t}^{i a}} d z-R \frac{n}{v} \int_{z_{0}}^{z}\left(\frac{1}{T_{t}^{i a}}-\frac{1}{T_{r}^{e f f}}\right) \\
& \times \sum_{i} \epsilon_{i} \sum_{j l m}\left(-P_{i} P_{j} k_{i j \rightarrow l m}+P_{\ell} P_{m} k_{\ell m \rightarrow i j}\right) d z,
\end{aligned}
$$

where the considerations given above for the transport coefficients in $\mathcal{D}^{(0)}$ are equally pertinent.

For computational convenience, the integrals in Eqs. (63) and (64) can be described by discrete sums over $z$, with sufficiently small $\Delta z$ discrete steps instead of $d z$. This option is favoured in practice by the fact that all experimentally derived quantities in Eqs. (63) and (64) can be described in tabular format.

\section{FINAL COMMENTS}

The present paper has been conceived as theoretical support for the experimental work at laboratories investigating supersonic jets, such as the Laboratory of Molecular Fluid Dynamics (CSIC, Madrid), in which, several $\mathrm{H}_{2}$ and $\mathrm{N}_{2}$ supersonic jets have been recently investigated, experimentally and by means of the equations reported here. ${ }^{63,64}$ Other jets involving small molecules whose collisions are of potential interest for different areas of science and technology will be studied in a short-medium term program.

Along the paper emphasis is made in the objectives declared in the Introduction, reporting a number of equations for those flow variables that cannot be measured accurately in the laboratory (translational temperature, velocity, dissipation, entropy) in terms of other variables more accessible to experiment (number density, rotational populations, and their gradients). These equations are Eq. (34) for the inviscidadiabatic temperature $\left(T_{t}^{i a}\right)$, Eqs. (54)-(56) for the isentropic temperature $\left(T_{t}^{i s e n}\right)$, and Eqs. (37) and (38) for the actual translational temperature $\left(T_{t}\right)$. Flow velocity in the inviscidadiabatic approximation $\left(v^{i a}\right)$ is given by Eq. (35). The actual flow velocity follows from Eq. (35), replacing $T_{t}^{i a}$ by $T_{t}$. Information on dissipation $(\mathcal{D})$ can be retrieved through Eqs. (19) and (36).

A description of entropy as a function of the abovementioned flow variables is given in Eqs. (41)-(43) in terms of dissipation $(\mathcal{D})$ and nonequilibrium $\left(T_{r}^{\text {eff }} \neq T_{t}\right)$. An alternative description in terms of number density $(n)$, translational temperature $\left(T_{t}\right)$, rotational populations $\left(P_{i}\right)$, and stagnation conditions is given by Eqs. (45)-(50).
Different ways how entropy can be interpreted are shown in Sec. IV, as well as the effect of entropy onto the actual translational temperature resulting from Eq. (57). A sample case shows in Fig. 1 the different entropic behavior in two jets at high and low exit Reynolds number $\operatorname{Re}_{e}$.

The reported equations, in connection to good-quality experimental data, provide a basis for the quantitative interpretation of paraxial flow in supersonic jets. In addition to the pure experimental interest, this may be of value for testing flow models and computational codes by means of jets characterized accurately.

Consequently, with the second goal of this work, the role of inelastic collisions in the n-LTE problem has been treated in the frame of Boltzmann equation (1) and generalized collision integral (2) by means of the kinetic master equation (6). In this context, the relevant equations are (39) and (63) for the translational temperature $\left(T_{t}\right)$, Eqs. (61) and (62) for the effective rotational temperature $\left(T_{r}^{\text {eff }}\right)$, and Eq. (64) for the entropy increment $\left(\Delta S^{(0)}\right)$. In these equations the $k_{i j \rightarrow \ell m}$ sts-rates [Laplace-transformed of the $\sigma_{i j \rightarrow \ell m}$ cross sections in the collision integral (2)] appear explicitly, quantifying the efficiency of the elementary collisions onto the macroscopic flow variables. The $k_{i j \rightarrow \ell m}$ sts-rates can be calculated today in the frame of quantum scattering methods by essentially the exact solution of a set of coupled Schrödinger equations. ${ }^{65}$ Experimental assessment for $k_{i j \rightarrow \ell m}$ rates has been possible in the low-temperature range for $\mathrm{H}_{2}: \mathrm{H}_{2}$, $\mathrm{N}_{2}: \mathrm{N}_{2}, \mathrm{O}_{2}: \mathrm{O}_{2}, \mathrm{H}_{2}: \mathrm{He}$ and $\mathrm{H}_{2} \mathrm{O}: \mathrm{He}$ systems by means of supersonic jet experiments ${ }^{66-71}$ combined with well-established quantum-scattering computational codes. ${ }^{72,73}$

Finally, since the calculated $k_{i j \rightarrow \ell m}$ collision sts-rates derive from the Schrödinger equation, high-quality experimental data together with Eqs. (61)-(64) might provide a way to test empirically the old controversial question of the physical compatibility of Schrödinger and Boltzmann equations. ${ }^{26}$

\section{ACKNOWLEDGMENTS}

This work has been supported by the Spanish Ministerio de Economía y Competitividad (MINECO) through the research Project Nos. FIS2013-48275-C2 and CONSOLIDER-ASTROMOL CSD2009-0038. Thanks are due to G. Tejeda for the $P_{i}$ and $n$ raw data on which Fig. 1 is based.

\section{APPENDIX: MEASUREMENT OF $z, n(z), P_{i}(z)$, AND $d P_{i}(z) / d z$ ALONG THE JET}

Since the pioneer works on jet diagnostics based on Raman scattering, ${ }^{5,74-78}$ a substantial progress has been possible thanks to the low-noise CCD-detectors, computer controlled multichannel spectrometers, ${ }^{79}$ and ad-hoc designed expansion chambers. ${ }^{39}$ Present state-of-the-art Raman spectroscopy has proved to be a powerful tool for the quantitative study of steady supersonic free jets. ${ }^{34,38,39,71,80,81}$ In its current stage it allows for accurate quantitative measurements under superb space resolution, at distances as close as $z / D=0.15$ to the orifice and up to $z / D \approx 20$ downstream in the jet. This is the region where the evolution of n-LTE can be observed best, with 


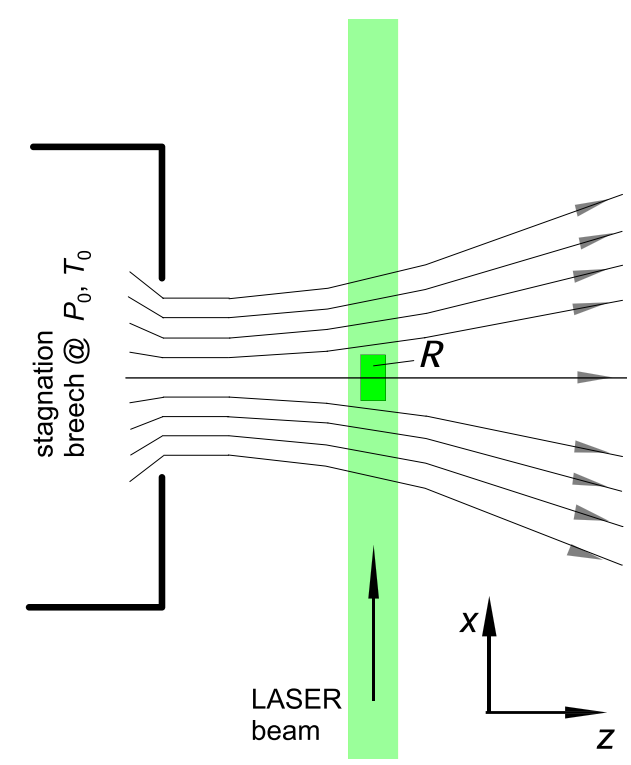

FIG. 2. Raman probing of a supersonic free jet. Raman scattering from $\mathcal{R}$ is collected along the $y$ direction.

dramatic cooling and rarefaction, and at which the equations given in this work are aimed. An outline of the experimental geometry is shown in Fig. 2, where $\mathcal{R}$ represents the gas parcel under scrutiny.

The width of $\mathcal{R}$ (along $z$ ) is controlled by the spectrometer slit, the depth (along $y$ ) by the waist of the sharply focused exciting laser beam, and the height (along $x$ ) by the readout system of the CCD-detector of the spectrometer. Probed volume $\mathcal{R}$ can be as small as $10 \times 15 \times 4 \mu \mathrm{m}^{3}$. No other experimental technique can reach such high resolution in the region close to the orifice. This space resolution allows for truly paraxial measurements. In addition to the high space resolution, Raman spectroscopy allows for remarkably accurate measurements of local number density $n(z)$, rotational (and in favorable cases also vibrational) populations $P_{i}(z)$, and gradients $d P_{i}(z) / d z$ in volume $\mathcal{R}$. These primary quantities are essential to work in the frame of the MEQ (6). As described in the main text, other local quantities like translational temperature $T_{t}(z)$, flow velocity $v(z)$, entropic increments $\Delta S(z)$, and time derivatives $d P_{i}(z) / d t$ can be obtained from the primary quantities. The primary quantities $z, n(z), P_{i}(z)$, and $d P_{i}(z) / d z$ are determined as follows.

\section{Distance $z$}

For technical reasons, the final section of the gas feeding line (gas breech and orifice) at stagnation conditions $p_{0}$ and $T_{0}$ is displaceable along $z$ while volume $\mathcal{R}$ remains fixed in the laboratory reference frame. Gas breech with orifice are mounted onto high-precision $\Delta x, \Delta y$, and $\Delta z$ motorized stages of $\pm 1 \mu \mathrm{m}$ positioning accuracy. The associated time resolution is on the order of nanoseconds.

Although the gas flows fast through $\mathcal{R}$, the molecules therein are continuously replaced by other molecules of identical statistical properties, and the system remains stationary by virtue of the ergodic principle. A number of measurement points at distances $z_{1}, z_{2}, \ldots, z_{m}$ are chosen along the jet axis in this way, and the local $n(z)$ and $P_{i}(z)$ quantities are measured there. Acquisition time for a single Raman spectrum at each $z$-point ranges from 30 to $150 \mathrm{~s}$. Up to twenty spectra per point are accumulated, depending on the intensity of the Raman scattering, which decreases fast with distance $z$.

\section{Number density $n(z)$}

Owing to the linear properties of Raman scattering, the number density at $\mathcal{R}$ can be measured from the integrated intensity $I_{v}(z)$ of a vibrational Raman Q-branch by means of the relation,

$$
I_{v}(z)=K \times n(z),
$$

where $K$ is a constant. Its value is determined measuring the intensity $I_{v}$ from a reference Raman spectrum recorded under static conditions of the gas (no expansion) at known number density in the chamber. Representative accuracy of $n(z)$ data in the jet are on the order of $2 \%$ near the orifice and about 5\% to $10 \%$ in the $z / D \approx 20$ downstream region.

\section{Rotational populations $P_{i}(z)$}

In jets with molecules of small rotational constants $(B<$ $3 \mathrm{~cm}^{-1}$ ) like $\mathrm{N}_{2}, \mathrm{O}_{2}, \mathrm{CO}, \mathrm{CO}_{2}$, and $\mathrm{N}_{2} \mathrm{O}$, the populations of rotational levels can be measured from the relative intensities of Raman lines of rotational transitions in the ground state. In jets with molecules of large rotational constants $\left(B>10 \mathrm{~cm}^{-1}\right)$ like $\mathrm{H}_{2}, \mathrm{H}_{2} \mathrm{O}$, and $\mathrm{CH}_{4}$ and their deuterated derivatives, the populations $P_{i}(z)$ are usually measured from the rotational lines of a vibrational band either from individual lines (like in $\mathrm{H}_{2}$ or $\left.\mathrm{CH}_{4}\right)^{69,77}$ or from the simulation of vibration-rotation unresolved band profiles (like in $\mathrm{H}_{2} \mathrm{O}$ ). ${ }^{71}$ Absolute values of $P_{i}(z)$ s can be routinely measured at $1 \%$ accuracy. Relative values of $P_{i}(z)$ s of several consecutive rotational levels can be as accurate as $0.1 \%$. In all cases, the populations $P_{i}(z)$ are obtained through the equation,

$$
I_{i \rightarrow j}(z)=K^{\prime}(z) \times f_{i j} \times g_{i j}(\alpha) \times P_{i}(z),
$$

where $I_{i \rightarrow j}$ are the relative intensities of $i \rightarrow j$ Raman lines; $K^{\prime}(z)$ is a constant at fixed $z ; f_{i j}=\left(v_{L}-\Delta v_{i j}\right)^{4}$ depends on exciting laser wavenumber $v_{L}$ and Raman shifts $\Delta v_{i j} ; g_{i j}$ is a quadratic function of the molecular polarizability $\alpha$, which is known for most molecular species of interest. At a fixed point $z$, the product $K^{\prime} \times f \times g$ is nearly constant in most cases, and the intensity $I_{i \rightarrow j}$ is proportional to the population of the initial state $i$ of the $i \rightarrow f$ Raman line. Absolute values of $P_{i}(z)$ follow from the normalization condition $\Sigma P_{i}=1$.

\section{4. $d P_{i} / d z$ gradients}

Sigmoidal functions of the type

$$
Y(z)=Y\left(z_{0}\right)+c \times \exp \left(-b /\left(z+z_{0}\right)^{a}\right),
$$

and polynomial combinations of them, provide highly accurate analytical descriptions for the $P_{i}(z)$ raw data; $z_{0}$ is the origin of the expansion, $Y\left(z_{0}\right)$ is the value of $P_{i}$ at the stagnation temperature $T_{0}$, and $a, b$, and $c$ are parameters fixed by a regression fit to the raw $P_{i}(z)$ data. The analytical derivation of such functions allows $d P_{i}(z) / d z$ data of up to $1 \%$ accuracy for $z<D$. 
${ }^{1}$ R. J. Gallagher and J. B. Fenn, "Relaxation rates from time of flight analysis of molecular beams," J. Chem. Phys. 60, 3487 (1974).

${ }^{2}$ L. K. Randeniya and M. A. Smith, "A study of molecular supersonic flow using the generalized Boltzmann equation," J. Chem. Phys. 93, 661 (1990).

${ }^{3}$ I. D. Boyd, "Rotational-translational energy transfer in rarefied nonequilibrium flows," Phys. Fluids A 2, 447 (1990).

${ }^{4}$ B. R. Cameron and P. W. Harland, "Monte Carlo calculation of rotational relaxation in small molecules," J. Chem. Soc., Faraday Trans. 89, 3517 (1993).

${ }^{5}$ I. D. Boyd, D. R. Beattie, and M. A. Cappelli, "Numerical and experimental investigations of low-density supersonic jets of hydrogen," J. Fluid Mech. 280, 41 (1994).

${ }^{6}$ A. Kantrowitz and J. Grey, "A high intensity source for the molecular beams. Part I. Theoretical,” Rev. Sci. Instrum. 22, 328 (1951).

${ }^{7}$ K. Bier and B. Schmidt, "Zur form der verdichtungsstõ $\beta$ e in frei expandierenden gas strahlen," Z. Angew. Phys. 13, 34 (1961).

${ }^{8}$ J. B. Anderson, R. P. Andres, and J. B. Fenn, "Supersonic nozzle beams," in Advances in Atomic and Molecular Physics, edited by D. Bates and I. Estermann (Academic Press Inc., New York, 1965).

${ }^{9}$ H. Ashkenas and F. S. Sherman, "The structure and utilization of supersonic free jets in low-density wind tunnels," in Proceedings of the 4th International Symposium on Rarefied Gas Dynamics, edited by J. H. deLeeuw (Academic Press, New York, 1966), Vol. 2, pp. 84-105.

${ }^{10} \mathrm{P}$. V. Marrone, "Temperature and density measurements in free jets and shock waves," Phys. Fluids 10, 521 (1967).

${ }^{11}$ E. P. Muntz, B. B. Hamel, and B. L. Maguire, "Some characteristics of exhaust plume rarefaction,” AIAA J. 8, 1651 (1970).

${ }^{12} \mathrm{~J}$. B. Anderson, "Molecular beams from nozzle sources," in Molecular Beams and Low Density Gas Dynamics, edited by P. P. Wegener (Marcel Dekker Inc., New York, 1974).

${ }^{13}$ M. J. Zucrow and J. D. Hoffman, Gas Dynamics (Wiley, New York, 1976), Vol. I.

${ }^{14}$ R. Campargue, "Progress in overexpanded supersonic jets and skimmed molecular beams in free jet zone of silence,"J. Phys. Chem. 88, 4466 (1984).

${ }^{15} \mathrm{H}$. R. Murphy and D. R. Miller, "Effects of nozzle geometry on kinetics in free jet expansions," J. Phys. Chem. 88, 4474 (1984).

${ }^{16}$ A. K. Rebrov, "Free jet as an object of non-equilibrium processes," in Proceedings of the 13th Symposium on Rarefied Gas Dynamics, edited by O. M. Belotserkovskii (Plenum Press, 1985), Vol. II, pp. 849-864.

${ }^{17}$ D. R. Miller, "Free jet sources," in Atomic and Molecular Beams, edited by G. Scoles (Oxford University Press, New York, 1988), Vol. I, p. 14.

${ }^{18}$ J. Anderson, Modern Compressible Flow: With Historical Perspective, 2nd ed. (McGraw-Hill, New York, 1990).

${ }^{19}$ B. Maté, I. A. Graur, T. Elizarova, I. Chirokov, G. Tejeda, J. M. Fernández, and S. Montero, "Experimental and numerical investigation of an axisymmetric supersonic jet," J. Fluid Mech. 426, 177 (2001).

${ }^{20}$ I. A. Graur, T. G. Elizarova, A. Ramos, G. Tejeda, J. M. Fernández, and S. Montero, "A study of shock waves in expanding flows on the basis of spectroscopic experiments and quasi-gasdynamic equations," J. Fluid Mech. 504, 239 (2004).

${ }^{21}$ A. Broc, S. DeBenedictis, G. Dilecce, M. Vigliotti, R. G. Sharafutdinov, and P. A. Skovorodko, "Experimental and numerical investigation of an $\mathrm{O}_{2} / \mathrm{NO}$ supersonic free jet expansion," J. Fluid Mech. 500, 211 (2004).

${ }^{22}$ P. A. Skovorodko, A. Ramos, G. Tejeda, J. M. Fernández, and S. Montero, "Experimental and numerical study of supersonic jets of $\mathrm{N}_{2}, \mathrm{H}_{2}$, and $\mathrm{N}_{2}+$ $\mathrm{H}_{2}$ mixtures," AIP Conf. Proc. 1501, 1228-1235 (2012).

${ }^{23}$ C. S. Wang-Chang and G. E. Uhlenbeck, University of Michigan Report No. CM-681, 1951.

${ }^{24}$ C. S. Wang Chang, G. E. Uhlenbeck, and J. de Boer, in Studies in Statistical Mechanics, edited by J. de Boer and G. E. Uhlenbeck (North-Holland, Amsterdam, 1964), Vol. 2.

${ }^{25}$ L. Waldmann, in Handbuch der Physik, edited by S. Flügge (Springer, Berlin, 1958), Vol. XII.

${ }^{26}$ R. F. Snider, "Relaxation and transport of molecular systems in the gas phase," Int. Rev. Phys. Chem. 17, 185 (1998).

${ }^{27}$ E. A. Mason and L. Monchick, "Heat conductivity of polyatomic and polar gases," J. Chem. Phys. 36, 1622 (1962).

${ }^{28} \mathrm{~L}$. Monchick and J. Schaefer, "Theoretical studies of $\mathrm{H}_{2}-\mathrm{H}_{2}$ collisions. II. Scattering and transport cross-sections of hydrogen at low energies: Tests of a new ab initio vibrotor potential," J. Chem. Phys. 73, 6153 (1980).

${ }^{29}$ W. E. Köhler and J. Schaefer, "Theoretical studies of $\mathrm{H}_{2}-\mathrm{H}_{2}$ collisions. V. Ab initio calculations of relaxation phenomena in parahydrogen gas," J. Chem. Phys. 78, 6602 (1983).
${ }^{30}$ F. R. W. McCourt, J. J. M. Beenakker, W. E. Köhler, and I. Kuscer, Nonequilibrium Phenomena in Polyatomic Gases (Oxford Science Publications, 1990, 1991), Vols. 1 and 2.

${ }^{31}$ V. M. Zhdanov, Transport Processes in Multicomponent Plasma (Taylor \& Francis, London, 2002).

${ }^{32}$ E. A. Nagnibeda and E. Kustova, Non-Equilibrium Reacting Gas Flows: Kinetic Theory of Transport and Relaxation Processes (Springer, Berlin, 2009).

${ }^{33}$ Those not confined by a nozzle in the initial section of the expansion.

${ }^{34} \mathrm{~S}$. Montero, "Raman spectroscopic experiments on Boltzmann collision integral in supersonic jets," AIP Conf. Proc. 1084, 3-11 (2008).

${ }^{35}$ H. Mori, T. Niimi, I. Akiyama, and T. Tsuzuki, "Experimental detection of rotational non-Boltzmann distribution in supersonic free molecular nitrogen flows," Phys. Fluids 17, 117103 (2005).

${ }^{36}$ S. Crist, P. M. Sherman, and D. R. Glass, "Study of highly underexpanded sonic jet," AIAA J. 4, 68 (1966).

${ }^{37} \mathrm{H}$. Alsmeyer, "Density profiles in argon and nitrogen shock waves measured by the absorption of an electron beam," J. Fluid Mech. 74, 497 (1976).

${ }^{38}$ G. Tejeda, B. Maté, J. M. Fernández-Sánchez, and S. Montero, "Temperature and density mapping of supersonic jet expansions using linear Raman spectroscopy," Phys. Rev. Lett. 76, 34 (1996).

${ }^{39}$ S. Montero, B. Maté, G. Tejeda, J. M. Fernández, and A. Ramos, "Raman studies of free jet expansion (diagnostics and mapping)," in Atomic and Molecular Beams: The State of the Art 2000, edited by R. Campargue (Springer, Berlin, 2001).

${ }^{40}$ H. Rabitz and S. H. Lam, "Rotational energy relaxation in molecular hydrogen," J. Chem. Phys. 63, 3532 (1975).

${ }^{41}$ H. Schlichting and K. Gersten, Boundary Layer Theory, 8th ed. (Springer, Berlin, 2000).

${ }^{42}$ S. Montero, "Temperature and entropy in supersonic free jets," Phys. Fluids 25, 056102 (2013).

${ }^{43}$ E. P. Muntz, "Measurements of anisotropic velocity distribution function in rapid radial expansions," in Proceedings of the 5th International Symposium on Rarefied Gas Dynamics, edited by C. L. Brundin (Academic, New York, 1967), Vol. II, pp. 1257-1286.

${ }^{44}$ R. Cattolica, F. Robben, L. Talbot, and D. R. Willis, "Translational nonequilibrium in free jet expansions," Phys. Fluids 17, 1793 (1974).

${ }^{45}$ A. K. Sreekanth, A. Prasad, and D. Prasad, "Numerical and experimental investigations of rarefied gas flows through nozzles and composite systems," in Proceedings of the 17th International Symposium on Rarefied Gas Dynamics, edited by A. E. Beylich (WCH, Weinheim, 1991), pp. 987-994.

${ }^{46} \mathrm{G}$. A. Bird, Molecular Gas Dynamics and the Direct Simulation of Gas Flows (Oxford University Press, Oxford, 1994).

${ }^{47}$ I. D. Boyd, G. Chen, and G. V. Candler, "Predicting failure of the continuum fluid equations in transitional hypersonic flows," Phys. Fluids 7, 210 (1995).

${ }^{48}$ R. A. Alberty, Physical Chemistry, 7th ed. (Wiley, New York, 1987).

${ }^{49}$ R. C. Tolman, The Principles of Statistical Mechanics (Dover, New York, 1979).

${ }^{50}$ T. L. Hill, Statistical Mechanics: Principles and Selected Applications (Dover, New York, 1987).

${ }^{51}$ G. H. Wannier, Statistical Physics (Dover, New York, 1987).

${ }^{52} \mathrm{~S}$. Chapman and T. G. Cowling, Mathematical Theory of Non-Uniform Gases (Cambridge University Press, Cambridge, 1970).

${ }^{53}$ J. O. Hirschfelder, C. F. Curtiss, and R. B. Bird, Molecular Theory of Gases and Liquids (Wiley, New York, 1954).

${ }^{54}$ W. A. Wakeham, "Transport properties of polyatomic gases," Int. J. Thermophys. 7, 1 (1986).

${ }^{55}$ C. Nyeland, L. L. Poulsen, and G. D. Billing, "Rotational relaxation and transport coefficients for diatomic gases: Computations on nitrogen," J. Phys. Chem. 88, 1216 (1984).

${ }^{56} \mathrm{~J}$. Schaefer, "Transport coefficients of dilute hydrogen gas, calculations and comparisons with experiment," Chem. Phys. 368, 38 (2010).

${ }^{57}$ H. W. Woolley, R. B. Scott, and F. G. Brickwedde, "Compilation of thermal properties of hydrogen in its various isotopic and ortho-para modifications," J. Res. Natl. Bur. Stand. 41, 379 (1948), paper RP 1932.

${ }^{58}$ M. J. Assael, S. Mixafendi, and W. A. Wakeham, "The viscosity and thermal conductivity of normal hydrogen in the limit of zero density," J. Phys. Chem. Ref. Data 15, 1315 (1986).

${ }^{59} \mathrm{~S}$. Montero and J. Pérez-Ríos, "Rotational relaxation in molecular hydrogen and deuterium: Theory versus acoustic experiments," J. Chem. Phys. 141, 114301 (2014).

${ }^{60} \mathrm{~S}$. Pascal and R. Brun, "Transport Properties in non-equilibrium gas mixtures,” Phys. Rev. E 47, 3251 (1993). 
${ }^{61}$ P. W. Hermans, L. J. F. Hermans, and J. J. M. Beenakker, "A survey of experimental data related to the non-spherical interaction for the hydrogen isotopes and their mixture with noble gases," Phys. A 122, 173 (1983).

${ }^{62}$ H. van Houten, L. F. J. Hermans, and J. J. M. Beenakker, "A survey of experimental data related to the non-spherical interaction for simple classical linear molecules and their mixtures with noble gases," Phys. A 131, 64 (1985).

${ }^{63}$ G. Tejeda, J. M. Fernández, and S. Montero, "Molecular description of para$\mathrm{H}_{2}$ and natural- $\mathrm{H}_{2}$ supersonic free jets," Phys. Fluids (to be published).

${ }^{64}$ F. Gámez, E. Moreno, G. Tejeda, J. M. Fernández, and S. Montero, "Molecular description of $\mathrm{N}_{2}$ supersonic free jets," Phys. Fluids (to be published).

${ }^{65}$ A. M. Arthurs and A. Dalgarno, "The theory of scattering by a rigid rotator," Proc. R. Soc. A 256, 540 (1960).

${ }^{66}$ B. Maté, F. Thibault, G. Tejeda, J. M. Fernández, and S. Montero, "Inelastic collisions in para- $\mathrm{H}_{2}$ : Translation-rotation state-to-state rate coefficients and cross sections at low temperature and energy," J. Chem. Phys. 122, 064313 (2005).

${ }^{67}$ S. Montero, F. Thibault, G. Tejeda, and J. M. Fernández, "Rotational state-to-state rates and spectral representation of inelastic collisions in low-temperature molecular hydrogen," J. Chem. Phys. 125, 124301 (2006).

${ }^{68}$ J. P. Fonfría, A. Ramos, F. Thibault, J. M. Fernández, and S. Montero, "Inelastic collisions in molecular nitrogen at low temperature $(2 \leq T \leq$ 50 K)," J. Chem. Phys. 127, 134305 (2007).

${ }^{69}$ G. Tejeda, F. Thibault, J. M. Fernández, and S. Montero, "Low-temperature inelastic collisions between hydrogen molecules and helium atoms," J. Chem. Phys. 128, 224308 (2008).

${ }^{70}$ J. Pérez-Ríos, G. Tejeda, J. M. Fernández, M. I. Hernández, and S. Montero, "Inelastic collisions in molecular oxygen at low temperature $(4 \leq T \leq$ $34 \mathrm{~K})$. Close coupling calculations versus experiment," J. Chem. Phys. 134, 174307 (2011).
${ }^{71}$ G. Tejeda, E. Carmona-Novillo, E. Moreno, J. M. Fernández, M. I. Hernández, and S. Montero, "Laboratory study of rate coefficients for $\mathrm{H}_{2} \mathrm{O}$ : He inelastic collisions between 20 and $120 \mathrm{~K}$," Astrophys. J., Suppl. Ser. 216, 3 (2015).

${ }^{72}$ J. M. Hutson and S. Green, MOLSCAT version 14, Collaborative Computational Project No. 6 of the UK Science and Engineering Research Council, 1994.

${ }^{73}$ D. R. Flower, G. Bourhis, and J.-M. Launay, "Molcol: A program for solving atomic and molecular collision problems," Comput. Phys. Commun. 131, 187 (2000).

${ }^{74}$ D. L. Hartley, "Transient gas concentration measurements utilizing laser Raman spectroscopy," AIAA J. 10, 687 (1972).

${ }^{75}$ W. D. Williams and J. W. L. Lewis, "Raman and Rayleigh scattering diagnostics of a two-phase hypersonic $\mathrm{N}_{2}$ flowfield," AIAA J. 13, 709 (1975).

${ }^{76}$ I. F. Silvera and F. Tommasini, "Intracavity Raman scattering from molecular beams: Direct determination of local properties in an expanding jet beam," Phys. Rev. Lett. 37, 136 (1976).

${ }^{77}$ G. Luijks, S. Stolte, and J. Reuss, "Molecular beam diagnostics by Raman spectroscopy," Chem. Phys. 62, 217 (1981).

${ }^{78}$ R. G. Shabram, A. E. Beylich, and E. M. Kudriavtsev, "Experimental investigation of $\mathrm{CO}_{2}$ and $\mathrm{N}_{2} \mathrm{O}$ jets using intracavity laser scattering," Prog. Astronaut. Aeronaut. 117, 168 (1989).

${ }^{79}$ G. Tejeda, J. M. Fernández-Sánchez, and S. Montero, "High performance dual Raman spectrometer," Appl. Spectrosc. 51, 265 (1997).

${ }^{80}$ A. Ramos, J. M. Fernández, G. Tejeda, and S. Montero, "Quantitative study of cluster growth in free-jet expansions of $\mathrm{CO}_{2}$ by Rayleigh and Raman scattering," Phys. Rev. A 72, 053204 (2005).

${ }^{81}$ A. Ramos, B. Maté, G. Tejeda, J. M. Fernández, and S. Montero, "Raman spectroscopy of hypersonic shock waves," Phys. Rev. E 62, 4940 (2000). 